\title{
AUTONOMOUS ROBOTIC DOZING FOR RAPID MATERIAL REMOVAL
}

*Gary M. Bone, Scott G. Olsen, and Graham E. Ashby 1280 Main St. W.

McMaster University,

Hamilton, Canada L8S $4 L 7$

(*Corresponding author: gary@mcmaster.ca) 


\title{
AUTONOMOUS ROBOTIC DOZING FOR RAPID MATERIAL REMOVAL
}

\begin{abstract}
The autonomous control of vehicles interacting forcibly with their environment, such as dozers and tractors, is an unsolved and challenging problem. Forces and motions are inherently coupled between the tool and the means of vehicle propulsion. Furthermore, they are often operated within uncertain and unstructured environments, such as those encountered in underground mining. There is a growing industrial interest in the development of robotic vehicles to improve productivity, efficiency and safety in mining and construction. This paper focuses on the modeling and control of autonomous robotic dozing for a material removal operation. A dozing process model has been developed based on observations of both a fullscale dozer and a scaled-down version. The model characterizes the dynamic interactions between the blade position, material accumulation on the blade, material distribution in the environment, and the motions of the dozer. The dozing control objective is to remove the loose material as rapidly as possible by driving forwards at full power while automatically raising/lowering the blade in response to sensor measurements. Two different controllers were developed to meet this objective. The first controller is based on a set of heuristic rules, and the second is an optimal controller based on the dynamic model. An instrumented scaled-down robotic dozer and dozing environment, designed to emulate the full-scale operation, are used to implement the controllers and compare their performances over multiple dozing passes. Experimental results are presented showing that the model-based controller increased the material removal rate by $33 \%$ compared to the rule-based controller. Lastly, technologies for full-scale implementation are discussed, followed by proposed future work and conclusions.
\end{abstract}

\section{KEYWORDS}

Autonomous dozer, mobile robots, dozing, hybrid dynamic systems, model-based control, optimal control, mining

\section{INTRODUCTION}

The use of autonomous systems is becoming more prevalent in industry due to their usefulness in improving safety, as well as reducing operational costs and cycle times. The construction and mining industries are no exception to this trend. The MineStar/MineGem systems developed by Caterpillar and the Automine system developed by Sandvik (Moore, 2006) are examples of automation systems that integrate mine operations and equipment management including condition monitoring autonomous machine control. It has been shown that equipment related accidents remain a significant safety concern in the mining industry. A study analyzing data from ten years of mining operations safety reports concluded that $37 \%$ to $88 \%$ of mine fatalities attributed to equipment related accidents (Kecojevic, Komljenovic, Groves, Radomsky, 2007). Thus, any degree of automation incorporating integrated safety systems can reduce risk to 
machines, operators and those working in close proximity. A number of innovative systems have been developed to enhance safety in mining operations. An overview of various safety oriented technology is presented in (Ruff, 2003).

The control of mobile machinery which interact forcibly with their environment, such as those needed in mining material removal, is a difficult, unsolved problem. The difficulty arises due to the inherent coupling of the tool (e.g., dozer blade) dynamics and those of the vehicle's means of propulsion (e.g., tracks). Moreover these machines generally operate in unknown and difficult terrain, adding to the difficulty.

An application where the resulting improvements in safety and productivity are expected to make the research and development efforts worthwhile is the use of autonomous robotic dozers to rapidly remove blasted material in a mining operation. An example of a manually operated low-profile dozer working in an underground mining environment is shown in Figure 1 (left). This application is well suited for robotic dozing due to the safety concerns. The operating environment is shown schematically in Figure 1 (right). Ore and rock material is blasted from the face into the panel where the dozer will operate. The dozer's goal is then to rapidly move this material into a larger, recessed section termed the gully. Material is later removed from the gully by some other machine or process, such as a load-haul-dump vehicle.
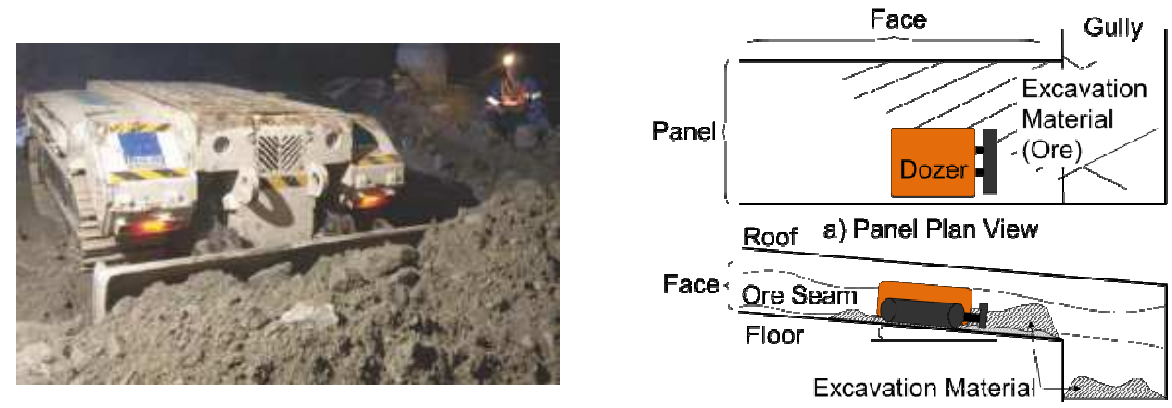

b) Panel Section View

Figure 1 - Left: A low profile dozer, shown with operator. Right: Schematic of the dozer and its operating environment

In this paper we present an overview of our work on the modeling and control of robotic dozing. It is important to note that prior research on the control of robotic dozing has either involved blade control for leveling soil, e.g., (Ito, 1991), or blade control to limit track slip, e.g., (Nakagami, 1997). Here the control objective is to move the loose material as rapidly as possible by driving forwards at full power while automatically raising/lowering the blade in response to sensor measurements. A dynamic model is developed and employed to create a model-based optimal controller. A rule-based controller is also described. The performances of the two controllers are compared experimentally using a scaled-down dozer and environment. This is followed by a discussion of technologies for full-scale implementation, and conclusions.

\section{DYNAMIC MODEL OF ROBOTIC DOZING}

A model of the dozing process dynamics is a pre-requisite for developing a high performance model-based controller. A dozer has two tracks, controlled by motors, and a dozing blade which can be raised and lowered via an actuator. The motors provide torque to the tracks which in turn applies a shear force to the material below the tracks. The magnitude of this force 
depends on the track/material friction as well as the geometry and arrangement of the material itself. Track slip can occur when the force required to move the material forward is greater than the traction forces, resulting in a slower speed of the dozer compared to the linear speed of the tracks. So there is a trade-off between the material accumulated on the blade and the forward speed of the dozer. The rate of material removal is the product of the amount of accumulated material and the dozer speed. Raising the blade will tend to decrease the amount of accumulated material while simultaneously increasing the speed (and vice-versa). This observation motivated our development of controllers whose objective is to maximize the material rate by raising/lowering the blade during each dozing pass.

During the dozing operation it is possible that the dozer could move in any of the six degrees-of-freedom (DOF); the blade could move along its one DOF; and the material could be arranged in some arbitrary three dimensional geometry. To focus on the primary low-level dynamic behavior, the model is kinematically constrained. The dozer's DOF are limited to X and $\mathrm{Z}$ motion, pitch, and the blade position; and the variations in the environment are primarily in the $\mathrm{X}$ and $\mathrm{Z}$ directions.

Observations of the full-scale dozing operation led to the conclusion that the overall operation could not be characterized by a single continuous dynamic model, and instead belongs to the class of hybrid dynamic systems (Branicky, Borkar, Mitter, 1998). Specifically, the dozing process can be decomposed into 10 distinct operational modes, each characterized by a continuous dynamic model, and 16 discrete mode transitions. Eight of these modes are illustrated in Figure 2. The set of equations representing the dynamics have the same structure with every mode, but their parameters change depending on the mode. The inputs to the system are: $r_{b}$, the commanded blade position; and the track control input. The outputs are the height of accumulated material, $d_{a}$ (see Figure 2d), the dozer speed, $v_{b}$, the pitch (see Figure $2 \mathrm{~g}$ ), the blade position, and the dozer's $\mathrm{X}$ and $\mathrm{Z}$ coordinates.

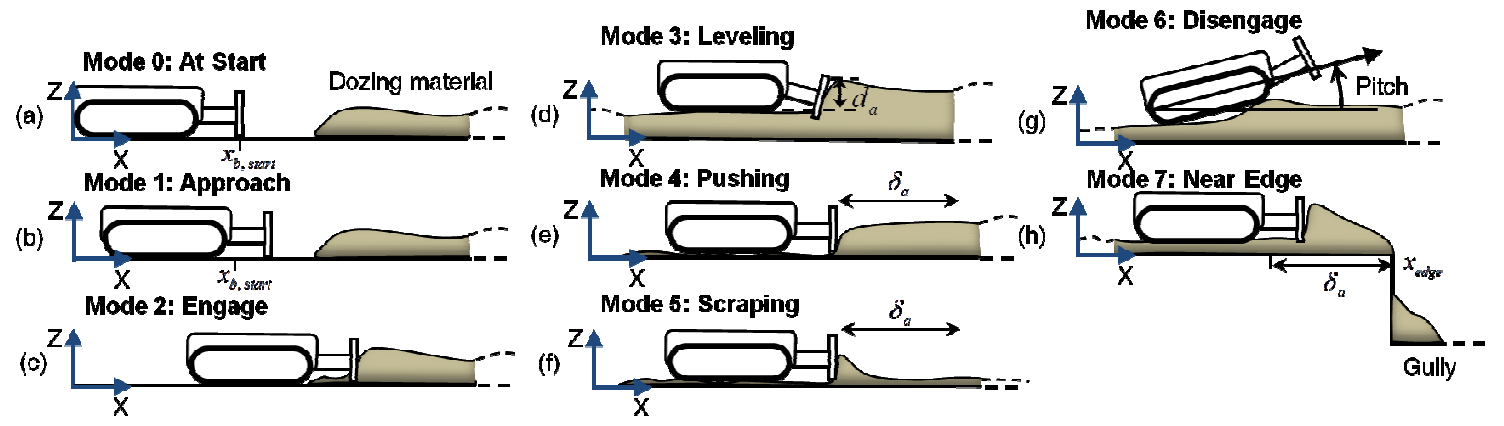

Figure $2-$ Schematic view of 8 of the 10 operational modes

These modes do not necessarily occur in numerical sequence as there are many mode transitions which depend on changes in the operational environment. Figure 3 shows all of the modes and mode transitions, denoted $\Sigma_{n}$. In the "At Start", "Approach", "Blade at Edge", and "Reverse" modes, the blade is servoed to a specified position. In the other modes the blade height is set by the controller. The modes are as follows:

- Mode 0: At Start - Dozer is at rest, in front of the leading edge of the dozing material. The blade is just touching the ground surface. See Figure $2 \mathrm{a}$. 
- Mode 1: Approach - The dozer moves forward. See Figure 2b.

- Mode 2: Engage - The dozer blade contacts the leading edge of the dozing material, and the dozer continues to move forward into the dozing material. Material accumulates on the blade, and the dozer tracks remains in contact with the floor surface (i.e., dozer elevation does not change). See Figure 2c.

- Mode 3: Leveling - If the dozing material is sufficiently high, the dozer climbs the pile. This loose material decreases traction. The blade can be lowered to penetrate down into the material pile, and accumulate material more rapidly. See Figure $2 \mathrm{~d}$.

- Mode 4: Pushing - The material pile is of negligible height at the blade, but of significant height ahead of the blade. The blade cannot penetrate further downward, so decreasing blade height will not increase accumulation, but will only increase resistance due to friction. See Figure 2e.

- Mode 5: Scraping - Similar to pushing, except that the material in front of the blade is also negligible in height. See Figure 2f.

- Mode 6: Disengage - The blade is raised while material is being pushed forward. Raising of the blade may cause material to form a localized mound, which the dozer will ascend until the blade loses contact with the material and no useful work is accomplished. See Figure 2g.

- Mode 7: Near Edge - When the blade is within a specified distance $\delta_{a}$ of the forward edge of the environment, material will be cast into the gully (see Figure $2 \mathrm{~h}$ ).

- Mode 8: Blade at Edge - The dozer has reached the forward edge of the operational environment, the track input is deactivated and the dozer stops.

- Mode 9: Reverse - The track input is specified to drive the dozer in reverse until it reaches the start position. The dozer blade is set to maximum height to avoid contact with material.

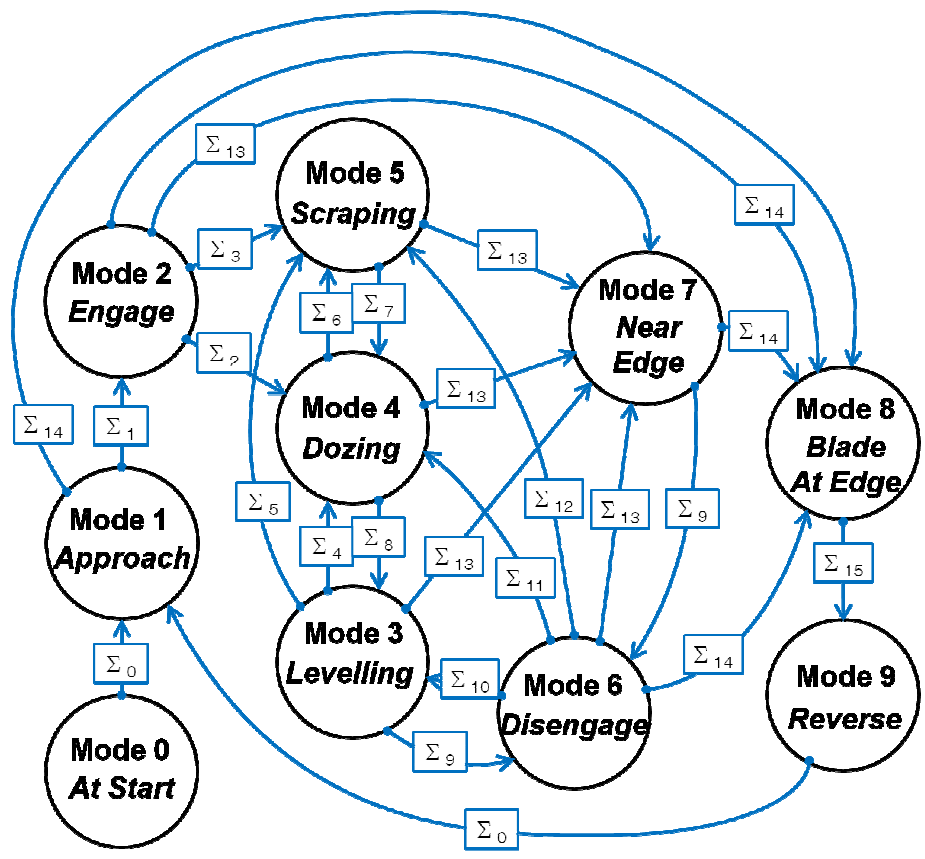

Figure 3 - Map of the 10 operational modes, and 16 mode transitions 
Clearing the requisite material can require multiple passes, that is to say the dozer can clear some material, reverse, and clear more the next pass. This cycle is repeated until all the specified material is removed, or the desired number of passes is reached. We define a single pass as the dozer beginning in mode 0 , transitioning through some variety of modes, and ending in mode 8 . We term a series of passes a trial. Full details of the model development are provided in (Olsen $\&$ Bone, 2013).

\section{CONTROLLER DEVELOPMENT}

Both a model-based optimal controller and a rule-based controller were developed to remove the material as quickly as possible. The rule-based controller has the advantages of requiring no system model, and being simpler to interpret and implement. This controller is described mathematically by the following algorithm:

$$
\begin{aligned}
& \text { 1. WHILE } x_{b}<x_{\text {edge }} \\
& \text { 2. } \quad \mathbf{I F}\left(d_{a}<d_{a \text { thresh }}\right) \wedge\left(v_{b} \geq v_{b, \text { thres }}\right) \wedge(\neg \mathrm{T} 1) \\
& \text { 4. } \quad r_{b}=r_{b}-\Delta r_{b} \\
& \text { 5. ENABLE T1 } \\
& \text { 6. ENDIF } \\
& \text { 7. } \quad \mathbf{I F}\left(v_{b}<v_{b, \text { thres }}\right) \wedge(\neg \mathrm{T} 2) \\
& \text { 8. } \quad r_{b}=r_{b}+\Delta r_{b} \\
& \text { 9. } \quad \text { ENABLE T2 } \\
& \text { 10. ENDIF } \\
& \text { 11. ENDWHILE }
\end{aligned}
$$

Where $x_{b}$ is the blade $\mathrm{X}$ position, $x_{\text {edge }}$ is the $\mathrm{X}$ position of the edge of the environment, $d_{a, t h r e s}$ is the material accumulation threshold, $v_{b, \text { thres }}$ is the dozer velocity threshold, T1 and T2 are delay-off timers, and $\Delta r_{b}$ is the commanded blade position increment. The delays T1 and T2 are used to avoid excessive change in blade position due to sensor noise.

A model-based optimal controller was developed next. Two different cost functions are proposed, one for mode 3 and one for modes 2, 4, 5 and 7 where blade control is required. These functions are necessary since we found that the rate of material accumulation is dependent on blade position in mode 3 , but not in the other controlled modes due to the constraint of the hard floor surface. The cost function to be minimized with mode 3 is:

$$
J_{3}=-\hat{d}_{a, k+1} \cdot \hat{v}_{b, k+1}+R_{3} \cdot r_{b, k}^{2}
$$

where $\hat{d}_{a, k+1}$ and $\hat{v}_{b, k+1}$ are the one-step ahead predictions of material accumulation and dozer velocity obtained from the measurements and hybrid dynamic model; and $R_{3}$ is the controller tuning parameter for mode 3 . The purpose of the first term in this equation is to maximize the predicted material removal rate, while the second term is included to allow the aggressiveness of the controller to be tuned. With the other modes the highest material removal rate is obtained when the blade maintains contact with the floor (i.e., minimizing $r_{b}$ ) while the dozer travels forward as fast as possible (i.e., maximizing $v_{b}$ ). The cost function that accomplishes this objective is: 


$$
J_{\text {modeNumber }}=\hat{v}_{b, k+1} \cdot r_{b, k}+R_{\text {modeNumber }} \cdot r_{b, k}^{2}
$$

with tuning parameter $R_{\text {modeNumber }}$. Note that the resulting equations for the commanded blade height, $r_{b, k}$, are functions of the model parameters and tuning parameter for the current operating mode. The model parameters were obtained from experimental data using system identification. Further details on the controller development are provided in (Olsen, 2012).

Note that the dynamics and control of the disengage mode (Mode 6) are a special case beyond the scope of the optimal control development framework. This mode is undesirable since it indicates that the dozer has lost contact with the underlying material, resulting in no bulldozing work being accomplished. The $d_{a}$ and $v_{b}$ dynamics of Mode 6 are fundamentally different from the other modes. Consequently, they could not be modeled within the same analytical structure of the system equations. It was found that Mode 6 avoidance can be accomplished with an appropriate state dependent blade position constraint imposed in Modes 2-5 and 7. The results of this investigation formed the basis for the development of a blade control law for Mode 6 to expedite a transition into a desirable mode (i.e. Mode 6 recovery). Further details on Mode 6 dynamics and control are also provided in (Olsen, 2012).

\section{EXPERIMENTAL SYSTEM}

The nature of full-scale experimental testing is impractical due to safety, cost and time for this type of machine and operation. As such, a scaled-down dozer and environment were developed to emulate the full scale operation. The dozer, shown in Figure 4 (left), combines a tracked vehicle with a dozer blade, position tracking target, and other sensors. The thrust is provided by two tracks driven by DC gearmotors. An actuator, consisting of a DC motor and lead screw, is used to move the blade. The blade measures $45 \mathrm{~mm}$ by $220 \mathrm{~mm}$. Above the blade, five optical range sensors measure the height of material accumulated on the blade relative to the base of the tracks. A tilt sensor measures the pitch of the dozer. Two tracking targets allow the position and heading to be measured at $16 \mathrm{~Hz}$ using an overhead camera. A second camera is used with a motor-driven laser stripe to measure the height of the material in the environment, before and after each dozing pass. The environment is composed of a $0.5 \mathrm{~m}$ by $2 \mathrm{~m}$ space containing loose material (stones $5 \mathrm{~mm}$ to $15 \mathrm{~mm}$ in size).
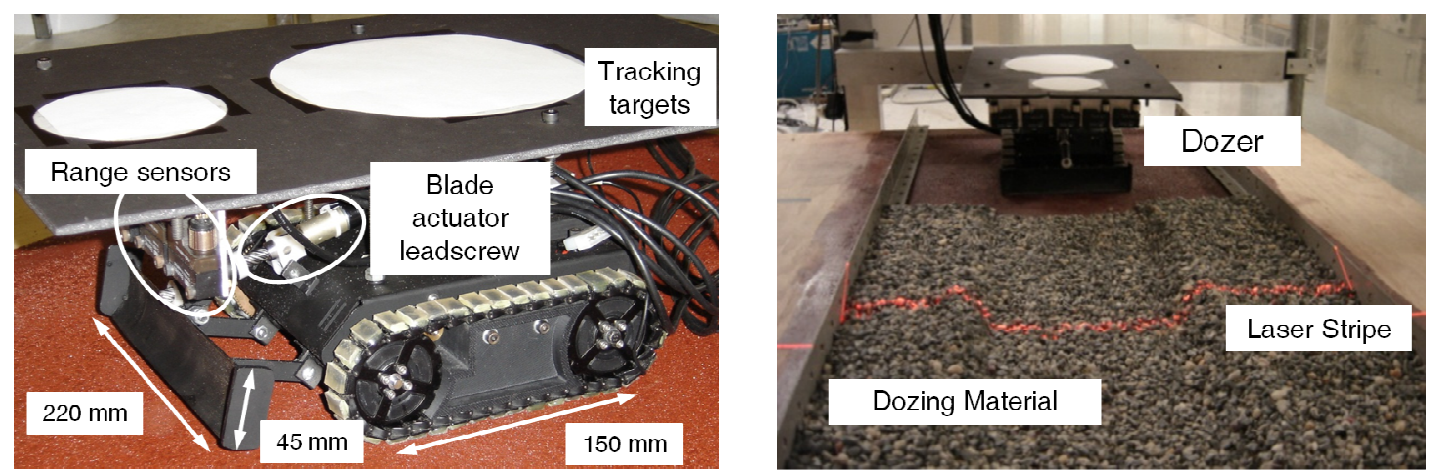

Figure 4 - Left: The scaled-down robotic dozer Right: The scaled down environment, with the scaleddown robotic dozer 


\section{EXPERIMENTAL RESULTS}

In order to evaluate the controllers, multi-pass trials were performed. The distribution of the material at the start of each trial had a height of $20 \mathrm{~mm}$ and a length of $1100 \mathrm{~mm}$. The material removal rate is $Q=d_{a} \cdot v_{b} \cdot w_{b}$, where $w_{b}$ is the blade width $(220 \mathrm{~mm})$. Eight trials (with 4 passes each) were performed. The comparison between the results of the optimal and the rulebased controller is shown in Figure 5. The per pass results averaged over the set of trials are indicated by the bar symbol. The improvement obtained by the model-based controller is most obvious in the first pass ( $37 \%$ increase in $\bar{Q}$ ), when the amount of material in the enviroment prior to the pass was greatest. As the amount of pre-pass material decreased, the distinction in the $\bar{Q}$ values between the controllers tended to diminish (i.e., 29\%, 43\% and 22\% increases after passes 2-4, respectively). Averaged over all of the passes and trials the model-based controller improved the material removal rate by $33 \%$.

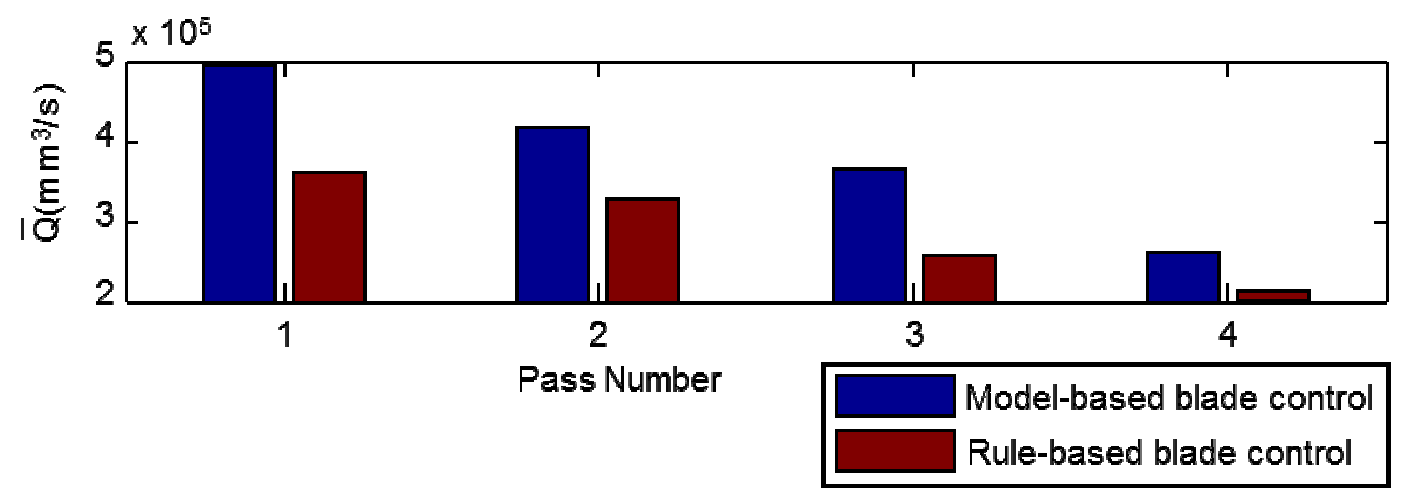

Figure 5 -Material removal rate for each pass, averaged over 8 trials

\section{TECHNOLOGIES FOR FULL-SCALE IMPLEMENTATION}

The current vision-based localization system and the vision-based laser scanning system are not intended for use as part of a full-scale system. For full-scale above-ground vehicle localization, global positioning system (GPS) based methods are becoming well-established for real-time vehicle localization and have been implemented on several heavy vehicles (Crane, Armstrong, Rankin, 1995), (Le, Rye, Durrant-Whyte, 1997) as well as consumer vehicles (Redmill, Kitajima, Ozguner, 2001). For underground applications various localization approaches have been investigated, including artificial beacons, inertial sensors, range finders and odometry (Scheding, Dissanayake, Nebot, Durrant-Whyte,1999), (Bakambu \& Polotski, 2007), and (Xiong, Han, Xiong, 2009). Material profile measurement for automation of mining and construction operations may be accomplished via sonar, or laser range finding, e.g., (Stentz, Bares, Singh, Rowe, 1999). Another alternative, well-suited for adverse environments, is millimeter wave radar (Brooker, Hennessey, Lobsey, Bishop, Widzyk-Capehart, 2007).

\section{FUTURE WORK}

Building on the results presented in this paper, there are a number of interesting avenues for extending this research. The overall experimental system scope could be extended to include different types of material for dozing, e.g. different sizes and densities of stones and/or soil; different blade shapes and sizes; and different floor surface textures. Furthermore, a more 
intensive investigation on track-slip could be conducted including detailed modeling and design of a control approach for track-slip reduction.

The scope of the bulldozing process could be extended to include additional degrees of freedom beyond the current constraints with a single direction of motion, e.g. introducing steep slopes to climb and introducing multi-directional planar navigation throughout the task space. In addition to low-level control design, higher-level strategies could be developed involving multiple bulldozing robots.

Different control laws could be formulated with different objectives other than to maximize the material removal rate. For example, a related bulldozing task involves blade control to achieve desired terrain profile characteristics for construction site preparation. This may entail formulation of a blade position control law with respect to minimizing the error between the actual underlying material profile height and a desired material profile height.

\section{CONCLUSIONS}

The development of a novel dozing process model and two novel controllers for autonomous robotic dozing have been presented. Based on observations of the full-scale dozing operation it was concluded that its dynamics belong to the class of hybrid dynamic systems. A model was then developed consisting of 10 distinct operational modes and 16 mode transitions. Next, a model-based controller and a rule-based controller were developed. The control objective for both is to remove the material as quickly as possible. The rule-based controller has the advantages of requiring no system model, and simpler implementation. However the modelbased controller uses optimization to provide superior performance. A scaled-down dozer and environment were developed to allow experiments to be performed in a safe and cost-effective manner. Eight four-pass dozing trials were conducted. Overall, the model-based controller increased the material removal rate by $33 \%$ compared to the rule-based controller. Technologies for full-scale implementation were also briefly reviewed.

\section{ACKNOWLEDGMENTS}

The funding provided by the Natural Sciences and Engineering Research Council of Canada is gratefully acknowledged.

\section{REFERENCES}

Bakambu, J.N., \& Polotski, V. (2007). Autonomous system for navigation and surveying in underground mines. Journal of Field Robotics, 24(10), 829-847. doi: 10.1002/rob.20213

Branicky, M.S., Borkar, V.S. \& Mitter,S.K. (1998). A unified framework for hybrid control: model and optimal control theory. IEEE Transactions on Automatic Control, 43(1), 31-45. doi: $10.1109 / 9.654885$

Brooker, G., Hennessey, R., Lobsey, C., Bishop, M., \& Widzyk-Capehart, E. (2007). Seeing through dust and water vapor: millimeter wave radar sensors for mining applications. Journal of Field Robotics, 24(10), 527-557. doi: 10.1002/rob.20166 
Crane, C. D., Armstrong, D. G., \& Rankin, A. L. (1995). Autonomous navigation of heavy construction equipment. Computer-Aided Civil and Infrastructure Engineering, 10(5), 357-370. doi: 10.1111/j.1467-8667.1995.tb00297.x

Ito N. (1991). Bulldozer blade control. Journal of Terramechanics, 28(1), 65-71. doi: 10.1016/0022-4898(91)90007-S.

Kecojevic, V., Komljenovic, D., Groves, W., \& Radomsky, M. (2007). An analysis of equipment-related fatal accidents in U.S. mining operations: 1995-2005. Safety Science, 45(8), 864-874. Doi:10.1016/j.ssci.2006.08.024

Le, A. T., Rye, D. C., \& Durrant-Whyte, H. F. (1997). Estimation of track-soil interactions for autonomous tracked vehicles. Proceedings of the 1997 IEEE International Conference on Robotics and Automation, 2, 1388-1393. doi: 10.1109/ROBOT.1997.614331

Moore, P. (2006). Return of the Robominers. Mining Magazine, 195, 16-22.

Nakagami, A. (1997). U.S. Patent No. 5,699,248. Washington, DC: U.S. Patent and Trademark Office.

Olsen, S. G. (2012). Modeling and control of three-dof robotic bulldozing (Doctoral dissertation). Retrieved from McMaster Open Access Dissertations and Theses. (Paper 7364)

Olsen, S. G., \& Bone, G. M. (2012). Development of a hybrid dynamic model and experimental identification of robotic bulldozing. Journal of Dynamic Systems, Measurement, and Control, 135(2). doi: 10.1115/1.4023061

Redmill, K. A., Kitajima, T., \& Ozguner, U. (2001). DGPS/INS integrated positioning for control of automated vehicle. Proceedings of the 2001 IEEE Intelligent Transportation Systems Conference, 172-178. doi: 10.1109/ITSC.2001.948650

Ruff, T.M. (2003). New technology to monitor blind areas near surface mining equipment. IEEE Industry Applications Conference, 38th Annual Meeting, 3, 1622 -1628. doi:10.1109/IAS.2003.1257773

Scheding, S., Dissanayake, G., Nebot, E. M., \& Durrant-Whyte, H. F. (1999). An experiment in autonomous navigation of an underground mining vehicle. IEEE Transactions on Robotics and Automation, 15(1), 85-95. doi: 10.1109/70.744605

Stentz A., Bares J., Singh A., \& Rowe P. (1999). A robotic excavator for autonomous truck loading. Autonomous Robots, 7(2), 175-186. doi: 10.1023/A:1008914201877

Xiong, C., Han, D., \& Xiong, Y., (2009). An integrated localization system for robots in underground environments. Industrial Robot, 36(3), 221 - 229. doi: 10.1108/01439910910950478 